\title{
CONTINUITY AND LOCATION OF ZEROS OF LINEAR COMBINATIONS OF POLYNOMIALS
}

\author{
MISHAEL ZEDEK ${ }^{1}$
}

1. Introduction. It is well known (see Marden [1, p. 3], Specht $\left[4\right.$, p. 13], Ostrowski [2]) that the zeros of a polynomial $\sum_{0}^{n} a_{k} z^{k}$ vary continuously when all its coefficients, with the exception of $a_{n}$, are varied continuously. The reason for this exception is that the situation is altered when the leading coefficient $a_{n}$ takes the value 0 . Theorem 1 is a formulation of a continuity principle where the leading coefficients are allowed to vanish and Theorems 3 and 4 apply this principle to the location of zeros of linear combinations of two polynomials. Theorem 4 improves on a recent result of Rubinstein [3]. Theorems 6 and 7 specify disks containing the zeros of a linear combination of a finite number of polynomials whose zeros lie in prescribed disks.

2. The continuity of zeros. The first theorem asserts that if the coefficients of a polynomial, some of whose leading coefficients are zero, are varied continuously, the existing zeros of the polynomial vary continuously whereas any new zeros emerge from a neighborhood of the point at infinity.

The coefficients of all polynomials in the following are complex numbers. $D(c, R)$ denotes the closed disk $|z-c| \leqq R$.

TheOREM 1. Given a polynomial $p_{n}(z) \equiv \sum_{0}^{n} a_{k} z^{k}, a_{n} \neq 0$, an integer $m \geqq n$ and $a$ number $\epsilon>0$, there exists a number $\delta>0$ such that whenever the $m+1$ complex numbers $b_{k}, 0 \leqq k \leqq m$, satisfy the inequalities

(1) $\left|b_{k}-a_{k}\right|<\delta$ for $0 \leqq k \leqq n$ and, if $m>n,\left|b_{k}\right|<\delta$ for $n+1 \leqq k \leqq m$, then the zeros $\beta_{k}, 1 \leqq k \leqq m$, of the polynomial $q_{m}(z) \equiv \sum_{0}^{m} b_{k} z^{k}$ can be labeled in such a way as to satisfy with respect to the zeros $\alpha_{k}, 1 \leqq k \leqq n$, of $p_{n}(z)$ the inequalities

(2) $\left|\beta_{k}-\alpha_{k}\right|<\epsilon$ for $1 \leqq k \leqq n$ and, if $m>n,\left|\beta_{k}\right|>1 / \epsilon$ for $n+1 \leqq k \leqq m$.

PRoof. We may assume that $\epsilon$ is sufficiently small so that the disks $D\left(\alpha_{k}, \epsilon\right), 1 \leqq k \leqq n$, are pairwise either identical or disjoint and that $D(0,1 / \epsilon)$ contains all the above disks. If $C_{k}$ denotes the boundary of

Presented to the Society, January 23, 1964; received by the editors September 6, 1963.

${ }^{1}$ Research supported by the National Science Foundation Grant No. G-24469 with the University of Maryland. 
$D\left(\alpha_{k}, \epsilon\right), 1 \leqq k \leqq n$, and $C_{0}$ is the boundary of $D(0,1 / \epsilon)$, then let $M=\min _{0 \leq k \leq n}\left(\min _{z \in C_{k}}\left|p_{n}(z)\right|\right)$. Obviously $M>0$. We pick $\delta$ to satisfy $\delta\left(\epsilon^{m}+\cdots+\epsilon+1\right)<M \epsilon^{m}$. If now the $b_{k}$ 's satisfy the inequalities (1), we have for $z \in U_{0}^{n} C_{k}$

$$
\left|q_{m}(z)-p_{n}(z)\right| \leqq \sum_{0}^{n}\left|b_{k}-a_{k}\right||z|^{k}+\sum_{n+1}^{m}\left|b_{k}\right||z|^{k} \leqq \sum_{0}^{m} \delta(1 / \epsilon)^{k}<M .
$$

Applying Rouché's theorem to $q_{m}(z)=p_{n}(z)+\left[q_{m}(z)-p_{n}(z)\right]$ for each of the circles $C_{k}, 0 \leqq k \leqq n$, we deduce that each of the disks $D\left(\alpha_{k}, \epsilon\right)$, $1 \leqq k \leqq n$, contains as many $\beta_{k}$ 's as $\alpha_{k}$ 's, thus implying the first part of (2), and that the disk $D(0,1 / \epsilon)$ contains precisely $n$ zeros of $q_{m}(z)$, which proves the second part of (2).

3. Linear combinations of two polynomials. Our purpose is to study the location of the zeros of the polynomial in $z F(z, \lambda) \equiv p_{m}(z)$ $+\lambda q_{n}(z)$ when it is known that the zeros of the polynomials $p_{m}(z)$ and $q_{n}(z)$ are, respectively, in the disks $D\left(c_{1}, R_{1}\right)$ and $D\left(c_{2}, R_{2}\right)$ and $\lambda$ is a constant. The special case $m=n$ was studied by Walsh [5]. We shall therefore assume for the moment $m \neq n$ and return to the special case in $\$ 4$. We first quote a theorem of Walsh [5]:

Theorem 2. If the points $\alpha_{1}, \alpha_{2}, \cdots, \alpha_{n}$ lie in the closed disk $D(c, r)$, the equation in $\alpha$

$$
\left(z-\alpha_{1}\right)\left(z-\alpha_{2}\right) \cdots\left(z-\alpha_{n}\right)=(z-\alpha)^{n}
$$

has at least one root in $D(c, r)$.

Next we prove a theorem about a special linear combination of polynomials:

Theorem 3. For a fixed $\lambda$, let $G(z, \lambda) \equiv\left(z-\alpha_{1}\right)^{m}+\lambda\left(z-\alpha_{2}\right)^{n}$, where $m$ and $n$ are arbitrary positive integers, $m>n \geqq 1$. Let $K=m^{m}\left|\alpha_{2}-\alpha_{1}\right|^{m-n} / n^{n}(m-n)^{m-n}$.

I. If $r_{1}$ is the unique positive root of the equation

$$
A(x) \equiv x^{m}-|\lambda|\left(x+\left|\alpha_{2}-\alpha_{1}\right|\right)^{n}=0,
$$

then the $m$ zeros of $G(z, \lambda)$ lie in the disk $D\left(\alpha_{1}, r_{1}\right)$.

II. If $|\lambda|>K$, let $r_{2}, r_{3}$ be the two ${ }^{3}$ positive roots of the equation

$$
B(x) \equiv|\lambda| x^{n}-\left(x+\left|\alpha_{2}-\alpha_{1}\right|\right)^{m}=0 .
$$

2 By Descartes' rule of signs and $A(0)<0, A(\infty)>0$.

- Since $B(0)<0, B(\infty)<0$, by Descartes' rule of signs there are at most two positive roots. We note that $B(y) \geqq 0$ is equivalent to $|\lambda| \geqq\left(y+\left|\alpha_{2}-\alpha_{1}\right|\right)^{m} / y^{m}=R(y)$ and that $K=\min [R(y), 0<y]$. 
Then $D\left(\alpha_{2}, r_{2}\right)$ contains exactly $n$ zeros of $G(z, \lambda)$ while the annulus $r_{2}<|z|<r_{8}$ contains none.

III. If $|\lambda|=K$, let $r_{2}$ be the unique positive (double) root of (4). Then at least $n$ zeros of $G(z, \lambda)$ lie in $D\left(\alpha_{2}, r_{2}\right)$, and at most $n$ zeros lie in its interior.

REMARK. It is easy to see that if for some $y_{1}>0$ we have $A\left(y_{1}\right)>0$, then $r_{1}<y_{1}$ and if for some $y_{2}>0$ we have $B\left(y_{2}\right)>0$, then $r_{2}<y_{2}<r_{3}$. Therefore $r_{1}$ and $r_{2}$ can be replaced by $y_{1}$ and $y_{2}$, respectively, in Parts I and II of Theorem 3. This would result in a weaker but more convenient proposition.

Proof. I. The $m$ zeros of $G(z, 0)$ are all located at $\alpha_{1}$. According to Theorem 1 , as we vary the values of the parameter continuously from 0 to $\lambda$, the same number of zeros of $G(z, \lambda)$ will lie in a disk $D\left(\alpha_{1}, y\right)$, $y>0$, unless at least one of the zeros, say $z^{\prime}$, has crossed the boundary $\left|z-\alpha_{1}\right|=y$ for some value $\lambda^{\prime}$ of the parameter. But for such $\lambda^{\prime}$ we have the inequality

$$
\left|\lambda^{\prime}\right|=\left|-\frac{\left(z^{\prime}-\alpha_{1}\right)^{m}}{\left(z^{\prime}-\alpha_{2}\right)^{n}}\right| \geqq \frac{y^{m}}{\left(y+\left|\alpha_{2}-\alpha_{1}\right|\right)^{n}} .
$$

Therefore if, for a fixed $y, \lambda$ satisfies the opposite inequality

$$
y^{m}-|\lambda|\left(y+\left|\alpha_{2}-\alpha_{1}\right|\right)^{n}>0,
$$

smaller values of $\lambda$ will also satisfy (6) and thus the $m$ zeros of $G(z, \lambda)$ are in the interior of $D\left(\alpha_{1}, y\right)$. Part I of our theorem follows from the observation that $y>r_{1}$ implies (6).

II. Set $\mu=1 / \lambda$ and $H(z, \lambda) \equiv G(z, \lambda) / \lambda=\left(z-\alpha_{2}\right)^{n}+\mu\left(z-\alpha_{1}\right)^{m}$. Again we observe that the $n$ zeros of $H(z, 0)$ are all at $\alpha_{2}$. By Theorem 1 , for sufficiently small values of $\mu$, the disk $D\left(\alpha_{2}, y\right), y>0$, would still contain exactly $n$ zeros of $H(z, \mu)$, the remaining $m-n$ zeros lying in a neighborhood of $\infty$. If for some value $\mu^{\prime}$ of the parameter, a zero, say $z^{\prime}$, of $H(z, \mu)$ is on the boundary of $D\left(\alpha_{2}, y\right)$, we would have

$$
\left|\mu^{\prime}\right| \equiv\left|-\frac{\left(z^{\prime}-\alpha_{2}\right)^{n}}{\left(z^{\prime}-\alpha_{1}\right)^{m}}\right| \geqq \frac{y^{n}}{\left(y+\left|\alpha_{2}-\alpha_{1}\right|\right)^{m}} .
$$

Therefore, if for a fixed value of $y, \mu$ satisfies the opposite inequali $\iota^{\prime}$

$$
y^{n}-|\mu|\left(y+\left|\alpha_{2}-\alpha_{1}\right|\right)^{m}>0,
$$

smaller values of $\mu$ will also satisfy (8), and thus $D\left(\alpha_{2}, y\right)$ will contain precisely $n$ zeros of $H(z, \mu)$, hence of $G(z, \lambda)$. Inequality (8) can be rewritten as $B(y)>0$ and it is seen easily that $r_{2}<y<r_{8}$ implies $B(y)>0$ and Part II follows immediately. 
III. If $|\lambda|=K, B\left(r_{2}\right)=0$ but for $0<y \neq r_{2}, B(y)<0$. However, if $|\lambda|>K$ (or $|\mu|<1 / K)$ we have

$$
r_{2}^{n}-|\mu|\left(r_{2}+\left|\alpha_{2}-\alpha_{1}\right|\right)^{m}>0
$$

which indicates that as the absolute value of the parameter grows from 0 to $1 / K$, the $n$ zeros of $H(z, 0)$ remain inside $D\left(\alpha_{2}, r_{2}\right)$ and the additional $m-n$ zeros, which appear when $\mu \neq 0$, remain outside $D\left(\alpha_{2}, r_{2}\right)$. Only when $|\mu|$ reaches the value $1 / K$ can some of the zeros of either group actually lie on the boundary of $D\left(\alpha_{2}, r_{2}\right)$. This completes the proof of Theorem 3 .

We now come to the main result of this section:

TheOREM 4. Let $f_{m}(z) \equiv z^{m}+a_{m-1} z^{m-1}+\cdots+a_{0}$ and $g_{n}(z) \equiv z^{n}$ $+b_{n-1} z^{n-1}+\cdots+b_{0}$ be two polynomials whose zeros lie, respectively, in the disks $D\left(c_{1}, R_{1}\right)$ and $D\left(c_{2}, R_{2}\right)$ and suppose $m>n \geqq 1$. For a fixed $\lambda$ let $F(z, \lambda) \equiv f_{m}(z)+\lambda g_{n}(z)$. Then:

I. If $\rho_{1}$ is the unique positive root of the equation

$$
C(x) \equiv x^{m}-|\lambda|\left(x+\left|c_{2}-c_{1}\right|+R_{1}+R_{2}\right)^{n}=0,
$$

then the $m$ zeros of $F(z, \lambda)$ lie in $D\left(c_{1}, R_{1}+\rho_{1}\right)$.

II. Setting $L=m^{m}\left(\left|c_{2}-c_{1}\right|+R_{1}+R_{2}\right)^{m} / n^{n}(m-n)^{m-n}$, the equation

$$
D(x) \equiv|\lambda| x^{n}-\left(x+\left|c_{2}-c_{1}\right|+R_{1}+R_{2}\right)^{m}=0
$$

has two positive roots $\rho_{2}, \rho_{3}\left(\rho_{2} \leqq \rho_{3}\right)$, provided $|\lambda| \geqq L$. At least $n$ zeros of $F(z, \lambda)$ lie in $D\left(c_{2}, R_{2}+\rho_{2}\right)$.

Remark. Again, as in Theorem 3, the above statements can be weakened but made more practical by replacing the disks $D\left(c_{1}, R_{1}+\rho_{1}\right)$ and $D\left(c_{2}, R_{2}+\rho_{2}\right)$ by the disks $D\left(c_{1}, R_{1}+y_{1}\right)$ and $D\left(c_{2}, R_{2}+y_{2}\right)$, where $y_{1}$ and $y_{2}$ satisfy the inequalities $C\left(y_{1}\right)>0$ and $D\left(y_{2}\right)>0$.

Proof. It follows from Theorem 2 that the zeros of $F(z, \lambda)$ are identical with the zeros of $G(z, \lambda) \equiv\left(z-\alpha_{1}\right)^{m}+\lambda\left(z-\alpha_{2}\right)^{n}$, where $\alpha_{1}$ and $\alpha_{2}$ are fixed, though undetermined, points in the disks $D\left(\alpha_{1}, R_{1}\right)$ and $D\left(\alpha_{2}, R_{2}\right)$, respectively. If we compare the equations (3) and (9) we see that since $\left|\alpha_{2}-\alpha_{1}\right| \leqq\left|c_{2}-c_{1}\right|+R_{1}+R_{2}$ we have $r_{1} \leqq \rho_{1}$. Similarly, comparing (4) with (10), we have $r_{2} \leqq \rho_{2} \leqq \rho_{3} \leqq r_{3}$. To conclude the proof it is sufficient to refer to Theorem 3 and to note that $D\left(\alpha_{1}, r_{1}\right) \subset D\left(c_{1}, R_{1}+\rho_{1}\right)$ and $D\left(\alpha_{2}, r_{2}\right) \subset D\left(c_{2}, R_{2}+\rho_{2}\right)$ regardless of the exact location of $\alpha_{1}$ and $\alpha_{2}$.

4. Linear combinations of several polynomials. As mentioned earlier, the case $m=n$ was studied by Walsh [5] who obtained the following result: 
ThEOREM 5. With the notations of Theorem 4 , if $m=n$, then the zeros of $F(z, \lambda) \equiv f_{n}(z)+\lambda g_{n}(z), \lambda \neq 1$, are in the union of the disks

$$
D\left(\frac{c_{1}-(-\lambda)^{1 / n} c_{2}}{1-(-\lambda)^{1 / n}}, \frac{R_{1}+\left.|\lambda|\right|^{1 / n} R_{2}}{\left|1-(-\lambda)^{1 / n}\right|}\right),
$$

where $(-\lambda)^{1 / n}$ takes all possible values. Any one of these $n$ disks which is external to all others contains precisely one zero.

The methods used in $\$ 3$ yield the following result which is also a corollary to Theorem 5 inasmuch as the disk in the corollary contains the $n$ disks in the theorem:

COROLLARY. With the notations of Theorem 4 , if $m=n$ and if $|\lambda|<1$, the zeros of $F(z, \lambda) \equiv f_{n}(z)+\lambda g_{n}(z)$ lie in the disk $D\left(c_{1}, R_{1}+\rho_{1}\right)$, where $\rho_{1}$ is the unique positive root of the equation

$$
x^{n}-|\lambda|\left(x+\left|c_{2}-c_{1}\right|+R_{1}+R_{2}\right)^{n}=0,
$$

that is,

$$
\rho_{1}=\frac{|\lambda|^{1 / n}\left(\left|c_{2}-c_{1}\right|+R_{1}+R_{2}\right)}{1-|\lambda|^{1 / n}} .
$$

Before turning to linear combinations of several polynomials of the same degree $n$, we establish a lemma which is of some interest in its own right:

LEMMA. Given the $k$ ( $\geqq 2)$ nonzero arbitrary complex numbers $\lambda_{1}, \cdots, \lambda_{k}$ which are subject to the conditions:

I. $\left|\lambda_{i}\right| \neq\left|\lambda_{j}\right|$ whenever $i \neq j$.

II. The sum of any number among the $\lambda$ 's is never 0 , we can always relabel the $\lambda$ 's so that

$$
\begin{aligned}
& \left|\lambda_{k}\right|<\left|\lambda_{k-1}+\cdots+\lambda_{1}\right|,\left|\lambda_{k-1}\right|<\left|\lambda_{k-2}+\cdots+\lambda_{1}\right|, \cdots, \\
& \left|\lambda_{3}\right|<\left|\lambda_{2}+\lambda_{1}\right|,\left|\lambda_{2}\right|<\left|\lambda_{1}\right| .
\end{aligned}
$$

Proof. The case $k=2$ is trivial. Suppose then that $k \geqq 3$ and that the lemma is valid for $2, \cdots, k-1$ complex numbers satisfying Conditions I and II. To prove our lemma by induction, it suffices to show that among the $k$ numbers there is one, say $\lambda_{k}$, which satisfies $\left|\lambda_{k}\right|<\left|\lambda_{k-1}+\cdots+\lambda_{1}\right|$. (The remaining inequalities in (13) will hold true by the assumption in the induction.) Indeed, otherwise, if $\left|\lambda_{i}\right| \geqq\left|S-\lambda_{i}\right|$ for $1 \leqq i \leqq k$, where $S=\sum_{1}^{k} \lambda_{i}$, squaring and adding the $k$ inequalities we would have $\sum_{1}^{k}\left|\lambda_{i}\right|^{2} \geqq \sum_{1}^{k}\left|S-\lambda_{i}\right|^{2}$ or $0 \geqq k|S|^{2}$ $-\sum_{1}^{k}\left(\lambda_{i} \bar{S}+\bar{\lambda}_{i} S\right)=(k-2)|S|^{2}$, which is false. 
THEOREM 6. Let $f_{i}(z) \equiv z^{n}+a_{n-1}^{(i)} z^{n-1}+\cdots+a_{0}^{(i)}, 1 \leqq i \leqq k$, be $k \geqq 2$ polynomials of the same degree $n$ whose zeros lie in the disks $D\left(c_{i}, R_{i}\right)$, respectively. Let $F\left(z ; \lambda_{1}, \cdots, \lambda_{k}\right) \equiv \lambda_{1} f_{1}(z)+\cdots+\lambda_{k} f_{k}(z)$, where the $\lambda_{i}$ 's are complex numbers satisfying Conditions I and II in the lemma and thus will be assumed to satisfy also the inequalities (13). Then the $n$ zeros of $F\left(z ; \lambda_{1}, \cdots, \lambda_{k}\right)$ are in the disk $D\left(c_{1}, R_{1}+\sum_{1}^{k-1} \rho_{i}\right)$ where the $\rho_{i}^{\prime}$ 's are determined recursively as follows: $\rho_{0}=0$, and for $1 \leqq i$ $\leqq k-1, \rho_{i}$ is the unique positive root of the equation

$$
x^{n}-\left|\lambda_{i+1} / \sum_{1}^{i} \lambda_{j}\right|\left(x+\left|c_{i+1}-c_{1}\right|+R_{1}+R_{i+1}+\sum_{0}^{i-1} \rho_{j}\right)^{n}=0 .
$$

Proof. The case $k=2$ follows from the corollary to Theorem 5 . We proceed with the proof by induction. Suppose our theorem holds true for a linear combination of $k-1$ polynomials. Then the zeros of $F\left(z ; \lambda_{1}, \cdots, \lambda_{k-1}\right)$ are in $D\left(c_{1}, R_{1}+\sum_{1}^{k-2} \rho_{i}\right)$ where the $\rho_{i}$ 's are determined again by (14). But

$$
\begin{aligned}
F\left(z ; \lambda_{1}, \cdots, \lambda_{k-1}, \lambda_{k}\right) & =F\left(z ; \lambda_{1}, \cdots, \lambda_{k-1}\right)+\lambda_{k} f_{k}(z) \\
& =\left(\lambda_{1}+\cdots+\lambda_{k-1}\right) g_{k-1}(z)+\lambda_{k} f_{k}(z),
\end{aligned}
$$

where $g_{k-1}(z) \equiv z^{n}+\cdots$ is a polynomial of degree $n$. Applying the corollary to the last linear combination of two polynomials of equal degree gives us $D\left(c_{1}, R_{1}+\sum_{1}^{k-1} \rho_{i}\right)$ as a disk containing the zeros of $F\left(z ; \lambda_{1}, \cdots, \lambda_{k}\right)$.

Theorem 6 permits us, except in some special cases, to replace by a single polynomial any group of polynomials of the same degree that appears in a linear combination of several polynomials. We shall therefore assume in our final theorem that the polynomials under consideration have distinct degrees:

THEOREM 7. Let $f_{i}(z) \equiv z^{n_{i}}+a_{n_{i}-1}^{(i)} z^{n_{i}-1}+\cdots+a_{0}^{(i)}, 1 \leqq i \leqq k, k \geqq 2$, be $k$ polynomials whose zeros lie in the disks $D\left(c_{i}, R_{i}\right)$, respectively. Suppose $n_{1}<n_{2}<\cdots<n_{k}$. Let $F\left(z ; \lambda_{1}, \cdots, \lambda_{k}\right) \equiv \sum_{1}^{k} \lambda_{i} f_{i}(z)$, where the $\lambda_{i}, s$ are arbitrary nonzero complex numbers. Then the $n_{k}$ zeros of $F\left(z ; \lambda_{1}, \cdots, \lambda_{k}\right)$ lie in the disk $D\left(c_{k}, R_{k}+\rho_{k}\right)$, where $\rho_{k}$ is determined recursively as follows: $\rho_{1}=0$, and for $2 \leqq i \leqq k, \rho_{i}$ is the unique positive root of the equation

$$
A_{i}(x) \equiv x^{n_{i}}-\left|\lambda_{i-1} / \lambda_{i}\right|\left(x+\left|c_{i}-c_{i-1}\right|+R_{i}+R_{i-1}+\rho_{i-1}\right) x^{n_{i}-1}=0 .
$$

REMARK. A remark similar to the ones made following Theorems 3 and 4 can be made here: in the statement of Theorem 7 the roots $\rho_{i}$ could be replaced by numbers $y_{i}>0$ for which $A_{i}\left(y_{i}\right)>0$. 
Proof. If we define the polynomials $h_{i}(z)$ recursively as follows: $h_{1}(z) \equiv f_{1}(z)$, and for $2 \leqq i \leqq k, h_{i}(z) \equiv f_{i}(z)+\left(\lambda_{i-1} / \lambda_{i}\right) h_{i-1}(z)$, we see that $F\left(z ; \lambda_{1}, \cdots, \lambda_{k}\right) \equiv \lambda_{k} h_{k}(z)$. Thus $h_{i}(z)$ is a linear combination of two polynomials to which Theorem 4 is applicable and the truth of Theorem 6 follows by induction.

\title{
REFERENCES
}

1. M. Marden, The geometry of the zeros of a polynomial in a complex variable, Math. Surveys No. 3, Amer. Math. Soc., Providence, R. I., 1949.

2. A. Ostrowski, Recherches sur la methode de Graeffe et les zeros des polynomes et des series de Laurent, Acta Math. 72 (1940), 99-257.

3. Z. Rubinstein, Analytic methods in the study of zeros of polynomials, Pacific J. Math. 13 (1963), 237-249.

4. W. Specht, Algebraische Gleichungen mit reelen oder komplexen Koeffizienten, Enzyklop. d. math. Wissen., 2nd ed., I 1, 3, II, Stuttgart, 1958.

5. J. L. Walsh, On the location of roots of certain types of polynomials, Trans. Amer. Math. Soc. 24 (1922), 163-180.

University OF MaryLand

\section{ZEROS OF EXPONENTIAL SUMS}

\author{
D. G. DICKSON
}

1. Introduction. This paper deals with the distribution in the complex plane of the zeros of an exponential sum of the form

$$
f(z)=\sum_{j=1}^{n} A_{j} z^{m_{j}}\left[1+\epsilon_{j}(z)\right] e^{\omega_{j} z},
$$

where $n>1$; the $A_{j}$ and the $\omega_{j}$ are complex numbers such that $A_{j} \neq 0$ and the $\omega_{j}$ are distinct; the $m_{j}$ are non-negative integers; the functions $\epsilon_{j}$ are analytic for $|z| \geqq r_{0} \geqq 0$ with $\lim _{z \rightarrow \infty} \epsilon_{j}(z)=0$. A function of the form

$$
\sum_{j=1}^{n} P_{j}(z) e^{\omega j z}
$$

Received by the editors September 9, 1963.

1 This work was supported by a grant from the National Science Foundation. 